\title{
RELACIÓN MÉDICO-PACIENTE: DERECHOS DEL ADULTO MAYOR
}

\author{
Melba Barrantes-Monge*, Eduardo Rodríguez**, Alexis Lama***
}

\begin{abstract}
Resumen: Existen prejuicios en relación con la vejez, incluso entre los profesionales que se dedican a la gerontología. Uno común y peligroso es considerar que los viejos son todos enfermos o discapacitados.

La relación médico-paciente es la piedra angular de la práctica y ética médicas. Para alcanzar el respeto por los adultos mayores es necesaria una medicina prudente, basada en una práctica en la cual la reflexión ética y clínica pueda contribuir. Esto último es posible si se hacen valer los derechos del adulto mayor, en particular como paciente para la toma de decisiones.
\end{abstract}

Palabras clave: bioética, geriatría, adulto mayor, consentimiento informado

\section{DOCTOR-PATIENT RELATIONSHIP: ELDERLY'S RIGHTS}

Abstract: Aging suffers prejudices against it. Even among professionals who practice gerontology. A dangerous and common one is to consider that all the elderly are sick or unable.

The relationship doctor-patient is the key stone both for medical practice and ethics. In order to achieve respect for the elderly a wise medicine is necessary, based upon a practice where ethical and clinical reflection may contribute. The latter is possible if the elderly's rights are respected, especially as patients when having to make decisions.

Key words: bioethics, geriatrics, elderly, informed consent

\section{RELAÇÃO MÉDICO-PACIENTE: DIREITOS DO IDOSO}

Resumo: Existem preconceitos em relação à velhice, inclusive entre os profissionais que se dedicam à gerontologia. É comum e perigoso considerar que os idosos são todos enfermos ou descapacitados.

A relação médico-paciente é a pedra angular da prática e ética médicas. Para se alcançar o respeito pelos idosos é necessária uma medicina prudente, baseada na prática que a reflexão ética e clínica podem contribuir. Esta última será possível se fizerem valer os direitos do idoso, em particular como paciente para a tomada de decisóes.

Palavras-chave: bioética, geriatria, idoso, consentimento informado

\footnotetext{
Médico. Oficina de Información Médica de la Dirección de Regulación Sanitaria, Ministerio de Salud. Nicaragua Correspondencia: melcruzbar@hotmail.com

** Doctor en Genética. Centro Interdisciplinario de Estudios en Bioética (CIEB), Universidad de Chile. Chile

*** Médico Cardiólogo. Diplomado en Bioética, Pontificia Universidad Católica de Chile. Chile
} 


\section{Introducción}

En la mayoría de los países americanos se experimenta un cambio demográfico debido a un incremento en la expectativa de vida y un descenso en la natalidad. Ejemplo de ello son Uruguay, Chile y México. En la actualidad, el $60 \%$ de personas mayores de 60 años viven en países en vías de desarrollo y se espera que para 2025 este porcentaje ascienda a un 75\%, de manera que tres de cada cuatro adultos mayores vivirán en estos países(1).

La vejez es una etapa normal de la vida: envejecemos desde que nacemos. En países en vías de desarrollo, se llama "ancianos", "adultos mayores" o "personas de la tercera edad" a quienes tienen 60 años o más, y en países desarrollados a los que tienen 65 años o más. En este artículo utilizaremos el término "adulto mayor".

Los adultos mayores corren el peligro de ser marginados y que se les prive de ejercer sus derechos. Esta situación de rechazo puede llevar a que se les considere personas no competentes, cuando quizás lo que han perdido es la autovalencia, pero sigue prevaleciendo su competencia y su capacidad mental para decidir los aspectos relacionados con su persona. Un clásico ejemplo es la "institucionalización", decisión tomada por familiares, en la mayoría de los casos los hijos, sin consultar al afectado.

Esa discriminación no sólo ocurre en lo social -con gran impacto sobre lo físico y mental de estas personas-, sino también en el campo de la salud.

Existen prejuicios hacia la vejez, vigentes en la sociedad, incluso en el discurso de los profesionales que se dedican a la gerontología. Entre estos prejuicios, el más común y peligroso es considerar que los viejos son todos enfermos o discapacitados.

Por lo antes expuesto, resulta necesaria una medicina prudente, donde prevalezca el respeto a la integridad del anciano. La reflexión ética y clínica puede contribuir en la práctica clínica geriátrica para atenuar la soledad y la impresión de inutilidad que a menudo presenta la persona de la tercera edad. Esto último permitirá que se mantenga la expresión de la personalidad y de la calidad de vida de la persona adulta, y mantener el sentido de su existencia(2).

\section{Principales problemas éticos}

La relación médico-paciente es la piedra angular de la práctica médica y, por lo tanto, de la ética médica(3). En la Declaración de Ginebra se exige al médico que debe "velar ante todo por la salud de su paciente" y el Código Internacional de Ética Médica estipula: "El médico debe a sus pacientes todos los recursos de su ciencia y toda su lealtad"(3). A pesar de lo anterior, es común que ocurra un deterioro en la relación médicopaciente en el grupo de los adultos mayores.

Si bien la información al paciente se ha ido introduciendo en la práctica sanitaria(4), en algunos casos ello no se hace directamente con éste sino con sus familiares. Algunos padecimientos que pueden generar esta situación son: deterioro cognitivo, enfermedad crónica incapacitante, pérdida de la autovalencia, dependencia económica, etc. Sin embargo, también puede deberse al ageísmo, viejismo o rechazo al anciano por parte del personal de salud, y sin ninguna de las condiciones previamente mencionadas. Butler (1993) definió este viejismo como "el prejuicio (que) se aplica principalmente (...) de la gente joven hacia la gente vieja". Salvarezza (2002) considera que el viejismo implica una conducta social compleja con dimensiones históricas, culturales, sociales, psicológicas e ideológicas, basada en un conjunto de prejuicios, estereotipos y discriminaciones que se aplican a los viejos en función de su edad. Quizás la empatía no se alcanza porque el personal sanitario no tiene la experiencia de haber sido viejo y, por lo tanto, presenta dificultad para ponerse en el lugar del otro, especialmente en una sociedad donde cada vez más los ancianos no conviven con la familia y, por lo tanto, llegan a ser personas desconocidas.

Es necesario apelar a los derechos de los adultos mayores no sólo en su calidad de tales, sino también como pacientes. Esto con el objetivo de mantener o mejorar su autonomía y calidad de vida (previniendo la discapacidad).

En la relación médico-paciente es habitual solicitar al personal de salud información diagnóstica, acompañada del pronóstico: resultado esperado del acto médico(5). En algunas ocasiones, este resultado obtenido no es exacto, debido al acto médico mismo; en otras, debido a la intervención de terceros (familiares o cuidadores). Esto último se torna problema cuando la información suministrada por el médico hacia el paciente es para conocer la situación, evolución y finalmente adoptar 
una forma de vida; o bien cuando el paciente tiene que colaborar con su tratamiento.

¿Qué sucede cuando la relación médico-paciente se encuentra intervenida por la familia? Resulta sencillo responder cuando se trata de pacientes que han perdido su competencia y capacidades mentales (el caso de las demencias en estadios severos), en los cuales existe dependencia total y está claramente establecido que el apoderado o familiares del paciente deben tomar las decisiones. Sin embargo, dicha situación se transforma en un conflicto ético en aquellos pacientes con dependencia funcional no severa y/o dependencia económica, pero con un nivel cognitivo íntegro.

No sería justo privar a los adultos mayores de algunos procedimientos diagnósticos y terapéuticos simplemente por ser "viejos", cuando el beneficio de mejorar la calidad de vida puede ser enorme. Otro acto de justicia consiste en escuchar al paciente, porque escuchar favorece la relación y la confianza. Uno de los motivos por los cuales el personal de salud rechaza atender al anciano es porque acude a la consulta con una lista de quejas. Es responsabilidad del profesional de salud permitir que el paciente relate y aclare sus quejas; esto le hace sentirse considerado $y$, como ocurre con frecuencia, no siempre la queja principal es el motivo de la consulta. Se debe cumplir también con un examen físico adecuado, así como con los estudios de laboratorios pertinentes. Este acto de justicia consiste en no privarlos de la tecnología cuando se toma en consideración el juicio de proporcionalidad.

Otro acto de justicia es permitir que los adultos mayores ejerzan sus derechos y, dentro de ellos, el ejercicio de su autonomía. Cualquier decisión del paciente es el resultado de su derecho de autonomía o autodeterminación, que viene impuesta por la naturaleza de los bienes jurídicos y por ser el paciente el portador de los mismos(5).

La autonomía consiste también en que, una vez emitida la decisión, no es necesaria la concurrencia de la voluntad de terceras personas. Esta situación es justificable únicamente cuando el adulto mayor no es capaz de tomar decisiones por alteración mental de algún tipo (es el caso de la demencia severa), y quienes deben tomar la decisión en este caso son los familiares $o$ apoderados.
A los pacientes mayores de edad con plena capacidad de prestar su consentimiento se les debe aplicar las reglas generales relativas al mismo. Estas reglas se centran en asegurar que la voluntad, tanto en su formación como en su manifestación, sea consciente y libre, siempre y cuando haya ausencia de vicios que lo afecten. Para que el consentimiento sea realmente adecuado debe cumplir con información adecuada, transmitir la información como un proceso continuo y notificar los riesgos previsibles (limitantes y excepciones).

Proporcionar información adecuada consiste en brindarla de modo que todos los adultos mayores tengan accesibilidad a ella. Para esto se deben considerar todas las limitantes del paciente, entre ellas las visuales y auditivas. Con el acceso a la información, el adulto mayor puede pensar en ella (pensamiento autónomo), pueda elegir su preferencia (pensamiento autónomo) y expresarla libremente (autonomía del deseo), para finalmente implementar su voluntad (autonomía en la acción). Otro acto de responsabilidad médica es asegurar de que el paciente comprenda toda la información relevante según sus necesidades y, en caso de algún impedimento, el facultativo está obligado a dar una dedicación especial. En ese momento la autonomía se convierte en un valor ético aún más crucial: cuando las condiciones de fragilidad y dependencia pueden amenazar la autodeterminación de los ancianos por el paternalismo de sus cuidadores $\mathrm{y} / \mathrm{o}$ familiares. Al adulto mayor se le debe advertir de los procedimientos diagnósticos y terapéuticos, así como del pronóstico y de los riesgos y beneficios de alguna medida emprendida o decisión tomada.

Documentos que comentan el tema de la investigación en personas vulnerables -como muchas veces son los ancianos- aceptan su inclusión siempre que(6):

- Los resultados de la investigación posean el potencial de producir beneficios directos y reales a la salud del probando.

- Una investigación de similar efectividad no pueda ser llevada a cabo en otro tipo de individuos competentes.

- La persona en que se investigará haya sido informada de sus derechos y las salvaguardas legales para su protección, en la medida en que es capaz de recibir la información.

- Se haya obtenido la autorización específica y documentada, por la autoridad o la representación legal de la persona. 
Excepcionalmente y cumplidos los puntos anteriores se podrá realizar un estudio cuyos resultados beneficien al probando o a otras personas de la misma categoría de edad o afectadas por la misma enfermedad o condición.

\section{Desacuerdo entre médico y adulto mayor}

Si el médico cree que el paciente ha hecho una elección inadecuada, por falta de conocimiento e información, está en la obligación de brindar información más completa y actualizada. Solamente si le parece que el individuo carece de capacidad suficiente (previos estudios adecuados) para entender la información y relevancia del problema médico, se debe solicitar un apoderado para proteger y asesorar al paciente o para que se tomen las decisiones correctas. Sin embargo, no podemos perder de vista que un paciente competente puede en cualquier momento abandonar el tratamiento, pero jamás el médico podrá abandonar al paciente. El adulto mayor es el único que puede autorizar algún tipo de intervención concerniente a su salud.

Éticamente, el médico no tiene obligación de prescribir un tratamiento elegido por el paciente cuando este tratamiento pone en riesgo su salud (situación que ocurre con frecuencia a esta edad: un ejemplo es la automedicación), aun si se trata de un paciente competente. En el caso de que estas diferencias no puedan ser resueltas, el médico puede retirarse del caso, siempre y cuando haya examinado los riesgos y beneficios de las opciones terapéuticas. De esta manera, el médico cumple con las normas sobre práctica e integridad profesional y se permite al paciente ejercer su autodeterminación.

Otro conflicto que puede ocurrir en la relación médicopaciente del adulto mayor es que a menudo en la práctica se crea un vínculo insano entre el profesional de salud (mayoritariamente los médicos) y su paciente anciano, en el cual el primero está persuadido que sólo él tiene todas las respuestas a la problemática de su paciente. ¿Cómo balancear el derecho a la autodeterminación del anciano y la firme creencia del profesional de que siempre habrá algo que hacer? $(7,8)$.

Por otra parte, en el tratamiento de las problemáticas de la tercera edad impera el "modelo médico", que implica centrarse únicamente en la cuestión orgánica individual y en su etiología fisiológica: se olvida el tratamiento de causas psicológicas y, fundamentalmente, de los efectos de las políticas sociales y económicas sobre la salud en el envejecimiento y en la vejez. Como consecuencia de esta ideología, los adultos mayores suelen centrar sus consultas en las dolencias físicas, manifestando pudor por expresar sus problemas psíquicos, tal vez por temor a ser estigmatizados.

Una de las mejores formas de respetar los derechos de los adultos mayores es que estos sean atendidos por médicos competentes, es decir, profesionales con algún grado de conocimiento de la psicología y biología de la vejez, tomando en cuenta los cambios sociales que estas personas experimentan. Lamentablemente, en muchos países del continente americano los adultos mayores son atendidos por médicos con poca o ninguna preparación en este aspecto. Hay países en los que ni siquiera existe una preparación mínima en pregrado. Por esto, es importante que las escuelas médicas latinoamericanas asignen en su malla curricular el tiempo suficiente a lo largo de toda la carrera para que el futuro médico tenga oportunidad de conocer la biología y los variados aspectos clínicos del envejecimiento, y desarrollar habilidades empáticas con los pacientes adultos mayores. Una mala atención y un mal tratamiento, además de afectar al enfermo, se torna en una carga social y económica para la familia y los sistemas de salud.

¿En qué momento y cómo debe involucrarse la familia en la relación médico-paciente de un adulto mayor competente?

Merece atención abordar aquellas situaciones en las cuales la familia de los pacientes capaces está también afectada por la decisión que ellos toman. Los vínculos de responsabilidad moral mutua en el núcleo familiar $\mathrm{y}$, sobre todo, en núcleos familiares tan extensos y compactos como los de países latinoamericanos, intervienen en el proceso de toma de decisiones. Esto es importante, principalmente en aquellas decisiones en las cuales se afecta seriamente la cantidad o calidad de vida del paciente, y en las cuales la familia asumirá sus cuidados. El problema resulta mayor cuando no es posible determinar el momento, la forma y el grado en que la familia debe tomar parte de la decisión. Sin embargo, de no ponerse condiciones en este sentido, la autonomía del paciente y su intimidad pueden correr serios peligros. Aunque si el paciente no acepta la participación de la familia y/o, por otra parte, el médico no facilita información, ambas posiciones pueden ser amenazantes para el propio paciente y para la familia. Posiblemente, la única solución sea efectuar 
acercamiento prudente y explícito, primero al paciente y luego a la familia, con el objeto de negociar sobre el problema en cuestión. En cualquier caso, es una cuestión abierta.

La toma de decisiones compartida requiere que el médico no sólo trate de satisfacer a su paciente, sino también de encontrar alternativas razonables, buscando en primer lugar el bien de su paciente y por ende de la familia. En el proceso de decisión compartida, el conocimiento del cuidador y la experiencia del facultativo pueden unirse con los valores más importantes para el adulto mayor; allí el bienestar del ser humano se reconcilia con el derecho individual de ser tratado como un sujeto y no como un objeto. El diálogo es una de las herramientas más importantes que el discurso bioético ha aportado a las sociedades modernas(9).

\section{Algunas normas y declaraciones para la relación médico-paciente del adulto $\operatorname{mayor}(10)$}

En la Declaración de Hong Kong de la Asociación Médica Mundial sobre el maltrato de los ancianos que se llevó acabo en la 41ª Asamblea Médica Mundial (septiembre, 1989), revisada en la 126a Sesión del Consejo Jerusalén (mayo, 1990) y en la 170a Sesión del Consejo de Divonne-les Bains en Francia (mayo, 2005) se adoptaron los principios generales para protección de los ancianos. Estos principios son:

- Los ancianos deben tener los mismos derechos a atención, bienestar y respeto que los demás seres humanos.

- El médico tiene la responsabilidad de ayudar a evitar el maltrato físico y psicológico de los pacientes ancianos.

- Si el médico es consultado por el anciano directamente, el hogar de ancianos o la familia, debe velar porque el paciente reciba la mejor atención posible.

- El médico que constate o sospeche de maltrato, como se define en esta Declaración, debe discutir la situación con los encargados, sea la familia o el hogar de ancianos. Si se confirma que existe maltrato o si se considera una muerte sospechosa, el médico debe informar a las autoridades correspondientes.

- Para garantizar la protección del anciano en cualquier ambiente, no debe haber restricciones a su derecho de elegir libremente el médico. Las asociaciones médicas nacionales deben luchar para que dicha libre elección sea respetada en el sistema médico-social.

La Asociación Médica Mundial también presenta las siguientes recomendaciones a los médicos que tratan a los ancianos y exhorta a todas las asociaciones médicas nacionales a dar publicidad a esta Declaración entre sus miembros y la opinión pública. Refiere que los médicos que atiendan ancianos deben(10):

- Hacer mayores esfuerzos para crear una relación de confianza con los pacientes ancianos, a fin de instarlos a pedir atención médica cuando sea necesario y a sentirse cómodos cuando confíen en el médico.

- Proporcionar una evaluación y tratamiento médico por los dańos producidos por el abuso y/o abandono.

- Intentar establecer o mantener una relación terapéutica con la familia (por lo general, el médico es el único profesional que mantiene un contacto duradero con el paciente y la familia) y mantener en la máxima medida posible la confidencialidad del paciente.

- Informar toda sospecha de casos de maltrato o abuso de ancianos, conforme a la legislación local.

- Utilizar un equipo multidisciplinario de tratantes de las profesiones médicas, servicio social, salud mental y legal, cada vez que sea posible, y

- Estimular la generación y utilización de recursos comunitarios de apoyo que presten servicios domiciliarios, reposo y disminución del estrés a las familias de alto riesgo.

La Declaración Universal de los Derechos Humanos, en los Pactos Internacionales de Derechos Humanos, así como el Plan de Acción sobre el envejecimiento establecen la necesidad de normas nacionales e internacionales para que los ancianos puedan gozar de protección.

Los derechos humanos fundamentales no disminuyen con la edad. Debemos estar convencidos de la marginación y los impedimentos que la vejez trae consigo. Por lo tanto, sería necesario generar una política para que jurídicamente este grupo de la población pueda tener protección y ejercer sus derechos. 


\section{Conclusión}

El anciano competente debe decidir en cuestiones de salud, según sus deseos y tras su consentimiento informado y voluntario, aunque la prudencia parece pedir un marco de toma de decisiones y responsabilidades compartido entre médico y anciano. Cuando el anciano es incompetente, la decisión se traslada a un familiar cercano que actúa como su representante. Si hay conflicto entre el adulto mayor competente y el médico o entre el médico y el representante familiar, el médico debe actuar siempre en el mejor beneficio del paciente. Una de las mejores formas de respetar los derechos de los adultos mayores es que estos sean atendidos por médicos competentes, es decir, profesionales con algún grado de conocimiento de la psicología y biología de la vejez, tomando en cuenta los cambios que estas personas experimentan.

\section{Referencias}

1. Zúńiga C, Vega E, Rodríguez Mańas L, et al. Propuesta de contenidos mínimos para los programas docentes de pregrado en Medicina Geriátrica en América Latina. Revista Panamericana de Salud Pública 2005; 17(5-6): 429-437.

2. Boitte P. El envejecimiento: Oportunidad para una medicina en busca de sus finalidades. Acta Bioethica 2001; 7(1): $9-25$.

3. Williams JR. Manual de Ética Médica. Francia: Asociación Médica Mundial; 2005: 14-60.

4. Lorda PS. El consentimiento informado. Abriendo nuevas brechas. En: Problemas prácticos del consentimiento informado. Barcelona: Víctor Grífols i Lucas; 2002: 11-61.

5. Romeo Casabona C. El consentimiento informado en la relación entre el médico y el paciente: aspectos jurídicos. En: Problemas prácticos del consentimiento informado. Barcelona: Víctor Grífols i Lucas; 2002: 63-133.

6. Kottow M. Marcos normativos de ética de la investigación cientifica con seres vivos. Santiago de Chile: CONICYT; 2007.

7. Daichiman LS. Controversia en los criterios éticos de los cuidados en geriatría. Acta Bioethica 2001; 7(1): 121-128.

8. Mercado Rodríguez C. Dilemas bioéticos en geriatría: toma de decisiones médicas. Acta Bioethica 2001; 7(1): 129142.

9. Lolas F. Las dimensiones bioética de la vejez. Acta Bioethica 2001; 7(1): 57-69.

10. Asociación Médica Mundial. Declaración de Hong Kong de la Asociación Médica Mundial sobre el maltrato de ancianos. Centro de Documentación de Bioética. Departamento de Humanidades Biomédicas; 2002. Sitio en Internet. Disponible en: http://www.unav.es/cdb/ammhongkong1.pdf

Recibido: 8 de abril de 2008

Aceptado: 17 de junio de 2008 